unknown-status partners in the prior 12 months. We defined three mutually-exclusive behavioural categories: serosorting (condomless anal intercourse [CAI] only with HIV-concordant partners); non-concordant CAI (any CAI with HIV-discordant/ unknown-status partners; [NCCAI]); and no CAI (consistent condom use or no AI). We estimated adjusted relative risks (aRR) between sexual behaviour and HIV/STI, and calculated the population attributable risk (PAR\%) as the proportion of HIV/STI cases attributable to serosorting (assuming serosorters would have otherwise consistently used condoms) and the population prevented fraction (PF) as the proportion of hypothetical HIV/STI cases averted by serosorting (assuming serosorters would have otherwise had NCCAI).

Results Behavioural data were complete for 49,912 visits; 91\% $(\mathrm{n}=45,220)$ were among self-reported HIV-negative MSM. Overall, serosorting was reported by $35 \%$ and $38 \%$ of HIV-negative and positive MSM, respectively. Compared to men reporting no CAI, HIV-negative serosorters had a significantly higher risk of HIV $(\mathrm{aRR}=2.0)$, syphilis $(\mathrm{aRR}=2.0)$, urethral gonorrhoea/chlamydia $(\mathrm{GC} / \mathrm{CT})(\mathrm{aRR}=1.5)$ and rectal GC/CT $(\mathrm{aRR}$ $=1.7$ ). The proportion of HIV/STI cases attributable to serosorting (PAR\%) were: HIV (15.3\%); syphilis (16.9\%); urethral GC/CT (11.9\%); rectal GC/CT (19.3\%). Serosorters had a significantly lower risk of $\mathrm{HIV}(\mathrm{aRR}=0.53)$ and syphilis $(\mathrm{aRR}=$ 0.76) compared to men reporting NCCAI; the proportion of HIV and syphilis cases averted by serosorting (PF) were $14.6 \%$ and $8.3 \%$, respectively. Among HIV-positive MSM, syphilis risk was significantly higher for serosorters compared to consistent condom users $(\mathrm{aRR}=1.4$; PAR\% $=12.1 \%)$.

Conclusion These data suggest that serosorting is responsible for, but also prevents, $15 \%$ of HIV cases among MSM (PAR $\%$ $=15.3 \%$ and $\mathrm{PF}=14.6 \%$ ), depending on the behaviour it replaces.

Disclosure of interest statement No pharmaceutical grants were received in the development of this study.

\section{P11.07 THE SPECTRUM OF SEXUAL BEHAVIOURS AMONG MSM AND ASSOCIATIONS WITH PREVALENT SEXUALLY TRANSMITTED INFECTIONS AND HIV}

${ }^{1} \mathrm{CE}$ Rice, ${ }^{1} \mathrm{AH}$ Norris, ${ }^{2} \mathrm{JA}$ Davis, ${ }^{3} \mathrm{CD}$ Lynch, ${ }^{4} \mathrm{KS}$ Fields, ${ }^{4} \mathrm{M}$ Envin, ${ }^{2} \mathrm{AN}$ Turner${ }^{*} .{ }^{1}$ Division of Epidemiology, College of Public Health, The Ohio State University, Columbus, OH, USA; ${ }^{2}$ Division of Infectious Diseases, Department of Internal Medicine, College of Medicine, The Ohio State University, Columbus, OH, USA; ${ }^{3}$ Department of Obstetrics and Gynecology, College of Medicine, The Ohio State University, Columbus, OH, USA; ${ }^{4}$ Sexual Health Clinic, Columbus Public Health, Columbus, OH, USA

\subsection{6/sextrans-2015-052270.455}

Introduction "Sex" among men who have sex with men (MSM) encompasses a much broader range of behaviours than just anal intercourse, yet most sexual health research in this population focuses on only this one practice. We undertook this study to more fully characterise the spectrum of sexual behaviours endorsed by MSM seeking care in an urban, public STD clinic and the associations between these behaviours and prevalent STI and HIV.

Methods This cross-sectional study of 235 MSM was conducted from July 2012 through October 2013. Participants self-administered a standardised survey assessing several categories of sexual behaviour, including multiple types of receptive/insertive behaviours, venues where sexual activity occurs, and sex while using drugs and alcohol. Men responded about behaviours over their lifetime and within the past three months. We generated unadjusted prevalence ratios (PRs) to characterise associations between recent participation in each behaviour and prevalent STI and HIV.

Results Participants' median age was 26 years. Most were white $(57 \%)$ and employed (73\%). Most, but not all, identified as gay $(76 \%)$. One-third $(35 \%)$ had rectal or urethral gonorrhoea or chlamydia, or primary/secondary syphilis. STI prevalence was significantly higher among men who reported using sex slings (PR: 2.04), anonymous sex (PR: 1.81), illicit drugs during sex (PR: 1.77), fisting (PR: 1.74), sex toys (PR: 1.44), and group sex (PR: 1.43). HIV prevalence was $17 \%$, and was significantly higher among men who endorsed fisting (PR: 4.81), felching (PR: 3.92), enemas (PR: 4.04), sex slings (PR: 2.47), drug use during sex (PR: 1.98), and group sex (PR: 1.92).

Conclusions Many behaviours other than anal intercourse were significantly associated with prevalent STI and HIV in unadjusted analyses, demonstrating the need for prospective studies to examine causal relationships between specific behaviours and STI or HIV acquisition.

Disclosure of interest statement No authors have any conflict to disclose.

\section{P11.08 KNOWLEDGE OF SEXUALLY TRANSMITTED DISEASE RISK AMONG MEN WHO HAVE SEX WITH MEN}

Salil C*, E Jeptanui. Department of Sexology, Information Centre for HIV Prevention, Moi Teaching and Referral Hospital, Eldoret, Kenya

\subsection{6/sextrans-2015-052270.456}

Introduction Sexually Transmitted Diseases (STDs) are the most frequent among Infectious diseases. In pathogenesis of the diseases that can be transmitted during sexual intercourse among, the most important is the problem of infected Anal secretions' transfer. That transfer may happen directly during the Anal's mucosa. The objective of the studies was risk awareness on STDs knowledge among men who have sex with men (MSM).

Methods To describe the problem, the studies were done on 50 men of which $60 \%$ were MSMs, and $30 \%$ were Bisexual. The survey was carried out using internet, through the web page for Homosexuals. The research was based on Personal Questionnaire; concerning demographical data, question about sexuality and knowledge of STD.

Results Almost half of the Men participating in the studies considered the level of their knowledge of STDs infection prevention were good. Most of them (ca.75\%) reported that has never suffered an STD before. About 52\% of the participants had problems to define infection risk in different sexual practices correctly, as well as to point out infection tracts. The biggest problem for them was to connect typical symptoms with particular diseases. However almost all participants assumed any STD's symptoms appearance is an indication for immediate medical attention.

Conclusions Although the prevailing parts of the participants were convinced to be well -informed about STDs, their knowledge was not deep - what can increase STDs infection risk. 